\title{
The Use of an Active Power Filter for Harmonic Elimination and Power Quality Improvement in a Nonlinear Loaded Electrical Installation
}

\author{
António P. Martins ${ }^{1}$ \\ ${ }^{1}$ Institute of Systems and Robotics - Porto \\ Faculty of Engineering - University of Porto \\ Rua Dr Roberto Frias, s/n, 4200-465, Porto, PORTUGAL \\ phone: +351 22 5081816; fax: +351 22 5081443; email: ajm@fe.up.pt
}

\begin{abstract}
Consumer electronics, domestic appliances and a large range of industrial applications, namely power electronics based, can cause high disturbances in the supplied electricity. In this paper, the power quality characteristics is addressed in terms of the related standards and in the possible measures to increase it. Going from single to three phase networks and discussing passive and active filtering solutions, the paper presents simulation and experimental results obtained with a three phase active filter prototype.
\end{abstract}

\section{Keywords}

Harmonic currents; Voltage distortion; Electrical installations; Active filters; Power quality

\section{Introduction}

The explosive growth in consumer electronics and domestic appliances has generated a major concern in the electricity supply industry, [1], [2]. Due to its circuit interface, these appliances draw current near the peak of the mains voltage. Usually, the circuit interface is a diode bridge rectifier followed by a large DC capacitor, as shown in Fig. 1.

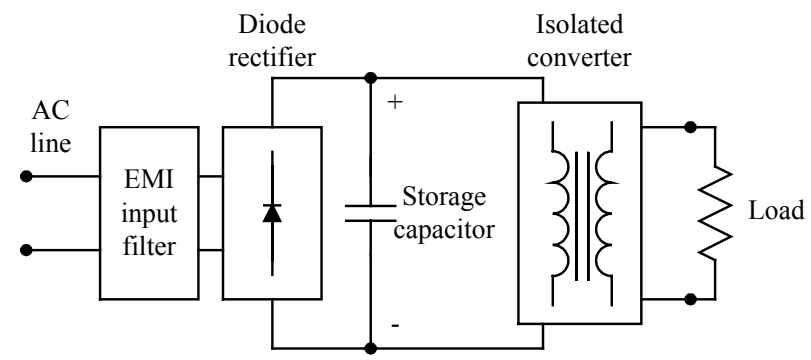

Fig. 1. Typical AC line interface for domestic appliances.

Like this circuit other power electronics based applications draw non-sinusoidal currents, despite the applied voltage being sinusoidal.
Examples of nonlinear loads include the following major examples:

- fluorescent lamps and phase-angle controlled lamp dimmers;

- the DC power supplies of any electronic product, whether linear or switch-mode;

- three-phase power converters;

- arc welding, electric furnaces, electrolytic processes, or other industrial applications like variable speed drives.

The harmonics generated by the most common nonlinear loads have the following properties:

- the lower order harmonics tend to dominate in amplitude;

- if the waveform has half-wave symmetry there are no even harmonics;

- the harmonic emissions from a large number of nonlinear loads of the same type will be added.

Small electronic devices are usually single phase loads, with only tens or hundreds of watts of mains power, but their proliferation are causing important effects in the mains voltage. Also, medium/high power nonlinear loads cause similar problems.

The major problems caused by the mains harmonic currents are those associated with the harmonic currents themselves, and those caused by the voltage waveform distortion resulting from the harmonic currents flowing in a finite supply source impedance, [3], [4].

This waveform voltage distortion can cause serious effects in direct on-line induction motors, ranging from a minor increase in internal temperature through excessive noise and vibration to actual damage; electronic power supplies may fail to operate adequately; increased earth leakage current through EMI filter capacitors due to their lower reactance at the harmonic frequencies. 


\section{Power quality and harmonic emissions standards}

With the increased use of electrical and electronic equipment, and the telecommunication and broadcasting transmissions the electromagnetic spectrum has become saturated. The equipment within domestic, commercial or industrial installations has become increasingly sensitive to some type of electromagnetic interference both from internal or external sources, primarily because of the use of digital technology. So there is a need for control of electromagnetic environment, namely by limiting of the harmonic emissions caused by any type of electrical or electronic equipment. In the European Union, this problem has been addressed by the 89/336/EEC Directive, (called the EMC Directive) which became into force in 1992. In the United States the main guideline come from the IEEE Std 519, [5].

The EMC Directive incorporates standards mainly from the CISPR, the CENELEC, and the IEC organizations. The standards assist in achieving adequate power quality and in controlling it. They provide a framework within which the supply environment, the susceptibility of equipment to low power quality, and the emissions from different types of equipment are all defined. Examples of standards relating to power quality characteristics and measurements are the EN 50160 and the IEC 61000-430 .

The purpose of the EN 50160 standard is to specify the characteristics of the supply voltage with regard to the course of the curve, the voltage level, the frequency and symmetry of the three phase network at the interconnecting point to the customer. The goal is to determine limiting values for regular operating conditions. However, facility defects may lead to major disturbances in the electricity distribution supply network. The complete breakdown of the network can no longer be described efficiently by limiting values. Thus there is no point in indicating actual limiting values. Accordingly, the standard establishes just these values as limiting values, which are not allowed to be exceeded or remained under during $95 \%$ of the controlled period. Rather than being an EMC standard the EN 50160 is a product standard giving the voltage characteristics which can be expected at the supply terminals. The EN 50160 standard is becoming a kind of reference for what should be seen as good power quality.

Like the EN 50160, the IEC 61000-4-30 is the first standard that defines the characteristics of voltage waveform as obtained from measurements. The power quality parameters considered are power frequency, magnitude of the supply voltage, flicker, supply voltage dips and swells, voltage interruptions, transients over voltages, supply voltage unbalance, voltage and current harmonics, voltage interharmonics and mains signalling on the supply voltage and rapid voltage changes. Depending on the purpose of the measurement, all of the phenomena on this list may be measured, or a subset of the phenomena on this list may be measured, [6], [7].
In this paper it is considered an electrical installation with particular characteristics: a very large number of PCs, printers, and faxes; fluorescent lamps, and laboratory instruments (oscilloscopes, DC power supplies, waveform generators, multimeters). The presence of low frequency harmonic currents demanded by the referred loads, the large number of inductive loads, [8], and the layout of the installation itself are the main cause of the poor power quality observed, [9].

The compatibility levels for low frequency conducted disturbances on low voltage networks are defined by IEC 61000-3-2.

\section{Low frequency harmonic currents and voltage distortion}

The problems caused by harmonic pollution can be divided into two categories:

- those caused by the harmonic currents themselves, and

- those caused by voltage waveform distortion resulting from the harmonic currents flowing in the electrical installation.

\section{A. Low frequency harmonics}

The main problem with the harmonic currents is that they can cause overheating in the local supply distribution transformer and in the installation itself. Also, harmonic currents in the neutral conductors of three-phase supplies present reliability and safety risks, where neutral conductors have not been suitably dimensioned. In Fig. 2 it is shown an example of the three-phase input current in a highly nonlinear loaded installation.

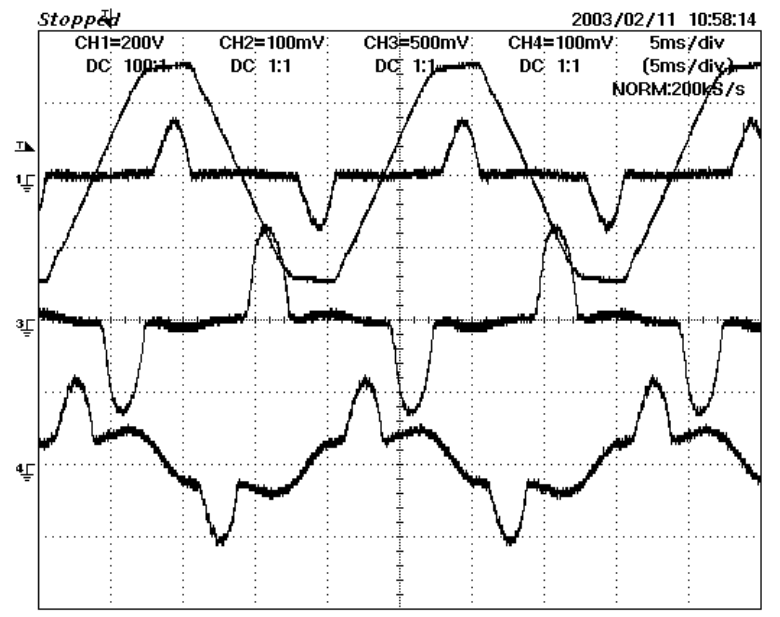

Fig. 2. Low frequency harmonics in a three phase installation.

The standards dealing with low frequency harmonic currents have evolved with the objective of promoting power quality by limiting the harmonic currents imposed on the mains supply. Examination of the recent changes to the standards show that they should have a positive effect in improving or at least maintaining power quality. The IEC 61000-3-2 incorporates four classes of equipment: A, B, D, and D. The Class D designation is 
for equipment having a "special wave shape" of input current and an active input power less than or equal to $600 \mathrm{~W}$, e.g. TVs, printers, and personal computer power supplies, except phase-angle-controlled motor driven equipment.

The "special wave shape" is defined by an envelope, shown in Fig. 3, which is effectively a means of distinguishing electronic power supply circuits, which normally draw their current for less than a third of the supply half-cycle. Equipment is deemed to be Class D if the input current wave shape of each half period is within the envelope shown for at least $95 \%$ of the duration of each half period.

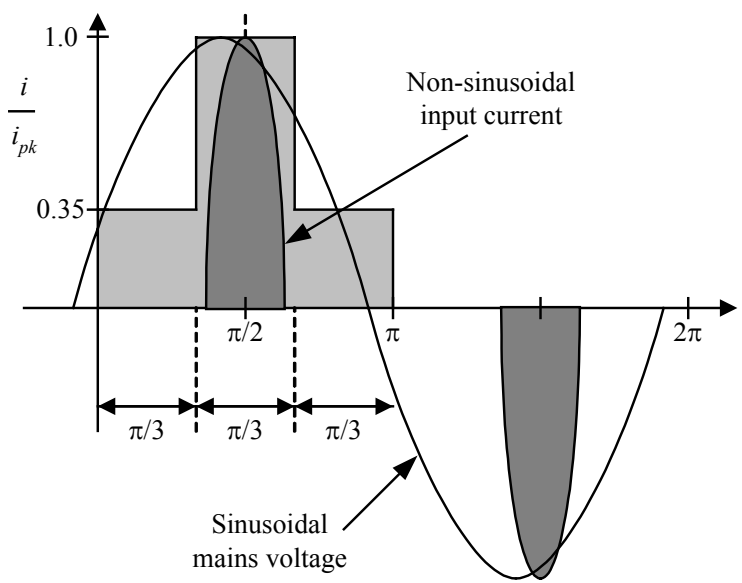

Fig. 3. Special wave shape for Class-D equipments.

\section{B. Mains signalling voltage and resonance}

The non-sinusoidal current drawn from the supply causes distortion of the supply voltage, since the inductance of the supply increases the source impedance as the harmonic order increases. This waveform distortion can cause serious effects in different loads. In this study it should be referred the malfunction associated with induction motors, electronic power supplies, and laboratory instruments.

System resonance effects at the harmonic frequencies can create areas of the power distribution network where the voltage is more heavily distorted than elsewhere, and/or has significant over- or under-voltage. Also, some areas of the network can suffer from much higher levels of current than elsewhere, at a few harmonic frequencies.

The signal transmission over the public network falls into one of two categories: ripple control systems (frequency range from $100 \mathrm{~Hz}$ to $3 \mathrm{kHz}$ ) and mains communication systems (frequency range from $3 \mathrm{kHz}$ to $148,5 \mathrm{kHz}$ ). The permissible voltage levels are given by EN 50160 (and in EN 61000-2-2). In an installation like the one described above the presence of these (limited) signalling voltages can produce resonance, the highest resonance occurring under low load conditions. In Fig. 4 it is shown an example of such phenomena, with a signalling resonance voltage of $14 \mathrm{~V}$ (in a $230 \mathrm{~V}, 50 \mathrm{~Hz}$ grid).

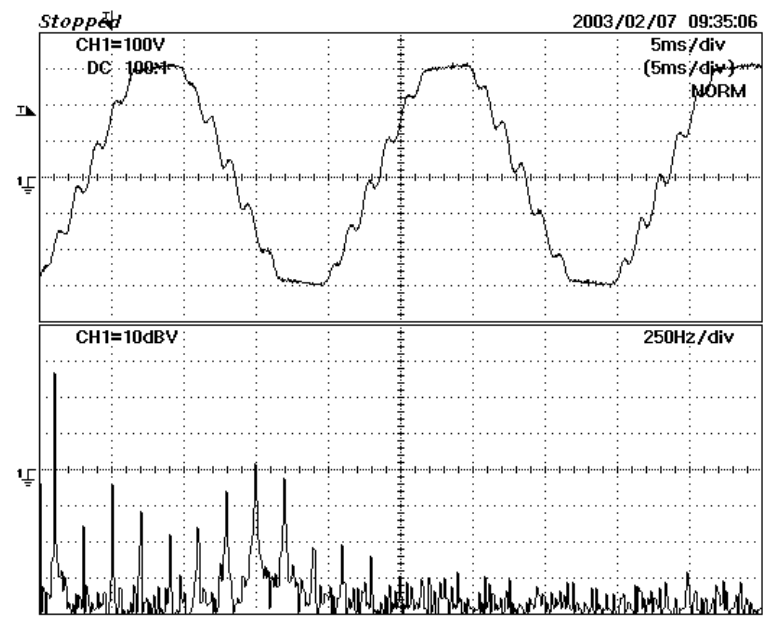

Fig. 4. Resonance in the presence of a mains signalling voltage at $750 \mathrm{~Hz}$.

\section{Active filtering solutions}

Different solutions to minimize the effects of nonlinear loads in electric distribution systems (non-sinusoidal voltages, harmonic currents, resonance) have been proposed and implemented. Even though the harmonic contribution of any overhead projector, Personal Computer, printer, or $200 \mathrm{~W}$ power supply, is negligible on its own, with hundreds of similar units connected to the same supply their contributions are additive and present a particular problem, depending on the capacity, impedance, and layout of the network.

\section{A. Low power interface}

In modern single phase low power equipments the mains interface is made with the circuit shown in Fig. 5. The converter in the interface can be controlled in different modes, [10]-[12], all of them achieving less harmonic distortion and higher power factor, namely changing the equipment classification which usually becomes Class A.

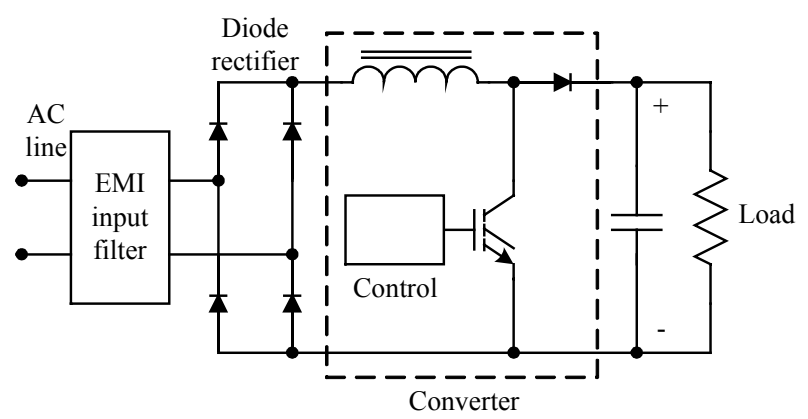

Fig. 5. Single-phase pre-regulation circuit with active filtering.

The main control categories are the line frequency switching compensators and the high frequency switching compensators. In the first control mode the line current conduction time is extended to almost the entire line period. High frequency switching solutions include the large family of power factor correction circuits, [12]. All of these ac/dc converter stages provide sinusoidal current absorption. In addition it should be referred 
various kinds of series and shunt compensators, like the active filters, [13]-[16 ].

\section{B. Medium/high power interface}

In three phase networks it is important to have balanced conditions and sinusoidal voltages. Also, it is desirable to achieve flexible AC power transmission systems, so incorporating power electronics circuits and other static compensators envisaging the increasing controllability and power transmission capacity, [13], [14].

Different types of compensators have been proposed to increase the electric system quality, [13]. One of those compensators is the voltage-source active power filter. In this paper it is used the parallel topology, schematically shown in Fig. 6.

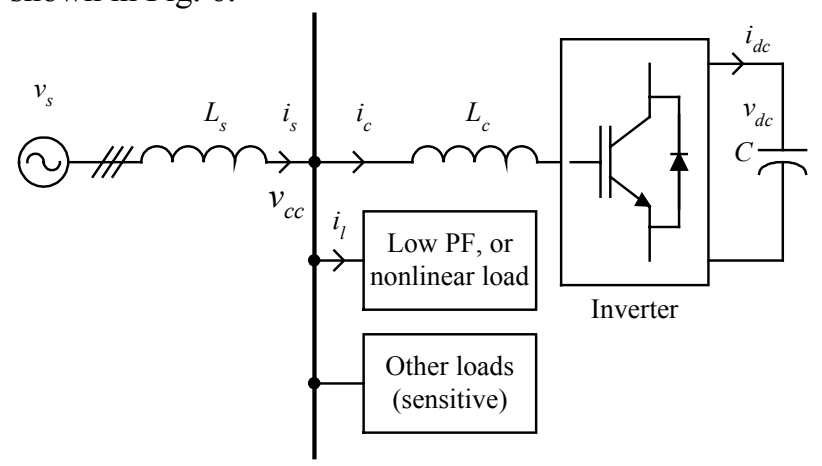

Fig. 6. Diagram of a voltage source active power filter.

The filter generates currents in the connection point in order to cancel the harmonic content in the AC system, and to correct the power factor. So, the AC distribution system only carries the active fundamental component of the load current, delivering an almost sinusoidal voltage at the connection point, $v_{c c}$.

\section{Active filter operation}

Very different control algorithms can be applied to the active filter, [15], [16]. The developed control system is based on a direct current control strategy that generates the reference waveform for the source current. It requires a low processing time and allows the calculation of the current reference for one of two strategies, [17]:

1- power factor correction, harmonic elimination, and load unbalance compensation, or

2- voltage regulation, harmonic elimination, and load unbalance compensation.

A $5 \mathrm{kVA}$ prototype of an active filter is designed, and tested in dynamic and stationary operation with different load types. A current-controlled pulse width modulation with a $5 \mathrm{kHz}$ fixed carrier ensures enough bandwidth to implement the active filtering. The control algorithm needs the measurement of several variables like the three phase AC source voltage and the DC link voltage. The active power balance in the DC link determines the reference current of the AC source and the use of a PI controller allows a smooth control of the filter current and improves the system dynamic response. The schematic in Fig. 7 represents the essential block diagram of the current reference.

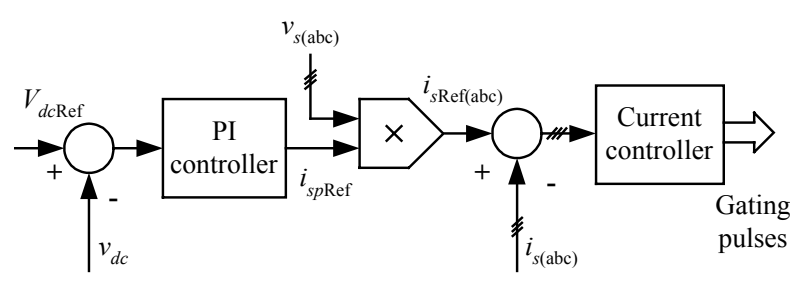

Fig. 7. Simplified block diagram for the calculation of the reference current.

The active filter control strategy is very similar to the one applied to the pre-regulator circuit in Fig. 5 in the high frequency switching mode with continuous current. The strategy implemented in this paper allows power factor correction to the unity, harmonic elimination and load unbalance compensation.

\section{A. Reference current determination}

The direct (or in-phase) unit current vectors are obtained from the instantaneous source voltages (assumed sinusoidal) and the rms amplitude of the source voltage, $V_{s p}$. For phase $a$, it results in (similar in the other phases):

$$
u_{s a}=v_{s a} / V_{s p}
$$

The amplitude of the active component of the reference currents of the AC source, $i_{s \operatorname{Ref}(\mathrm{abc})}$, is calculated through a PI controller with anti-windup as shown in Fig. 7. The proportional and integral gains determine the controller behaviour in dynamic and static operation. As stated before the correct active component, $i_{s p \text { Ref }}$, of the $\mathrm{AC}$ source current is determined from the power balance in the DC link. The current reference is then scaled with the unit current vectors in phase with the source voltage. For phase $a$, it results in:

$$
i_{s \operatorname{Ref}(\mathrm{a})}=i_{s p \operatorname{Ref}} \cdot u_{s a}
$$

In the modulation stage the total reference currents are subtracted from the source current, obtaining a current error adapted according to the amplitude of the triangular carrier. The purpose of introducing the triangular waveform is to stabilize the converter switching frequency by forcing it to be constant and equal to the frequency of the triangular reference signal. Since the current error signal is always kept within the negative and positive peaks of the triangular waveform, the system has an inherent over current protection.

\section{B. Simulation and experimental results}

The SABER simulation software package was used to design, simulate, and test the control algorithm. In order to implement the algorithm in the hardware platform it was built a simulation model for the microcontroller. So, the $\mathrm{C}$ code written and validated in SABER can be easily transferred to any hardware target. 
The simulation transient operation tests were made first connecting the filter and then connecting the load under test. In the case of Fig. 8 the load is a three phase diode rectifier.

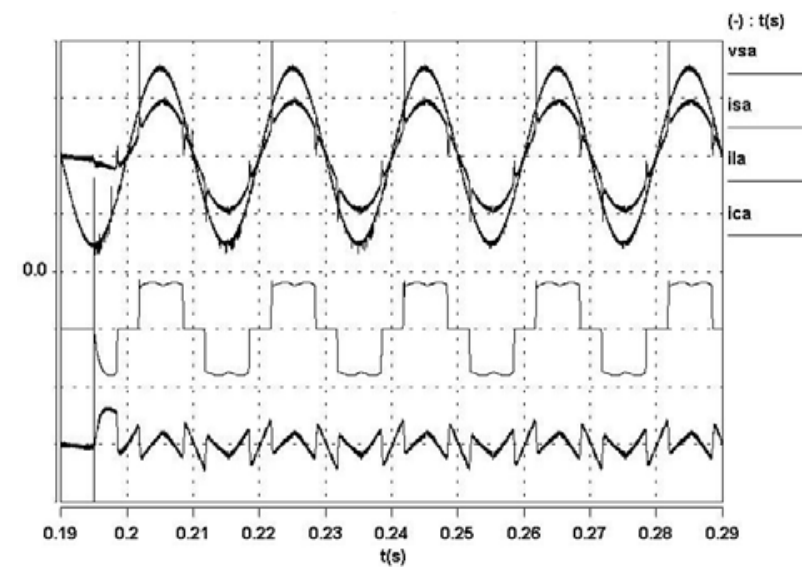

Fig. 8. Dynamic compensation of a three phase diode rectifier. Traces from top to bottom (phase A): source voltage, $v_{s a},(100$ $\mathrm{V} / \mathrm{div})$; source current, $i_{s a},(10 \mathrm{~A} / \mathrm{div})$; load current, $i_{l a},(10$ $\mathrm{A} / \mathrm{div})$; filter current, $i_{c a},(10 \mathrm{~A} / \mathrm{div})$.

As a demonstration of the filter experimental operation, the nonlinear load shown in Fig. 9 is again a three phase diode rectifier. It can be seen that the mains current gets a sine waveform, being only slightly distorted in the instants of the diode switching. This is due to the high $d i / d t$ occurring in these points, which is impossible to compensate unless with a very high DC voltage or a very low AC inductance. There should be a compromise between the active filter dimensioning and the characteristics of the load current to be compensated.

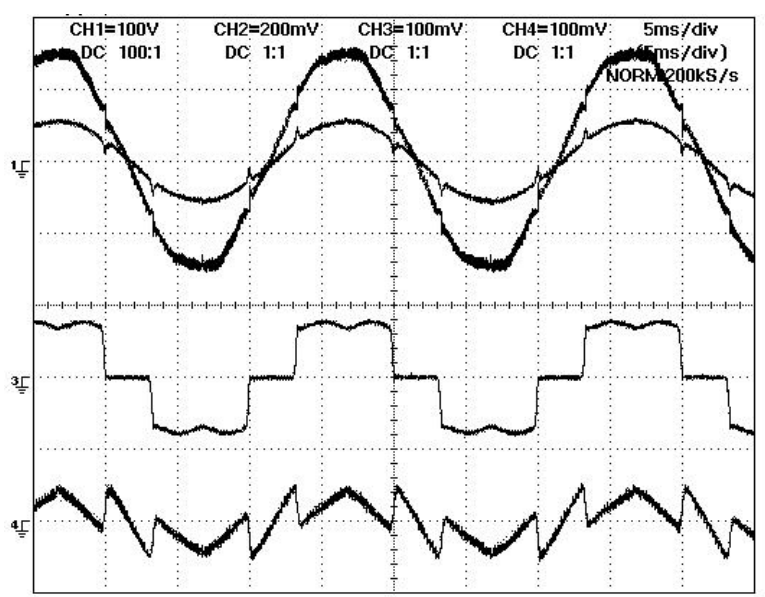

Fig. 9. Compensation of a three phase diode rectifier. Traces from top to bottom (phase A): source voltage, $v_{s a},(100 \mathrm{~V} / \mathrm{div})$; source current, $i_{s a},(20 \mathrm{~A} / \mathrm{div})$; load current, $i_{l a},(10 \mathrm{~A} / \mathrm{div})$; filter current, $i_{c a},(10 \mathrm{~A} / \mathrm{div})$.

\section{Conclusions}

Harmonics generated by nonlinear loads are one of the major causes of a poor power quality. So, harmonic elimination, in the source or with active filtering, is needed to achieve a better power quality. The paper addresses the problem of active filtering in low power single phase networks and medium/high power three phase networks.

In modern single phase low power equipments they should have a pre-regulator stage, achieving an almost sinusoidal input current. In medium/high power single or three phase networks when it is not possible to eliminate the harmonic currents in the input stage in some connection point, the active power filter is the solution to be implemented. The active filter operation in the harmonic elimination mode allows an increasing in the power quality due to the achieved sinusoidal current flowing in the network.

The presented results obtained with a three phase active filter prototype show its effectiveness both in static and dynamic operation, namely with a high nonlinear load.

\section{References}

[1] EURELECTRIC, "Power Quality in European Electricity Supply Networks", Brussels, (2002).

[2] M. H. J. Bollen, "Understanding Power Quality Problems", IEEE Press, Piscataway, (2000).

[3] J. Arrillaga, D. A. Bradley and P. S. Bodger, "Power Systems Harmonics", John Wiley and Sons, Chichester, (1985).

[4] C. Sankaran, "Power Quality”, CRC Press, Boca Raton, (2002).

[5] IEEE Std 519-1992, "IEEE Recommended Practices and Requirements for Harmonic Control in Electric Power Systems", IEEE Press, Piscataway, (1993).

[6] J. A. Oliver, R. Lawrence and B. B. Banerjee, "Power Quality", IEEE Industry Applications Magazine, Sept/Oct. 2002, pp. 21-30.

[7] R. P. Rico, "Calidad de Potencia. La Calidad del Servicio Eléctrico en Redes Públicas de Baja y Media Tensión", Mundo Electrónico, No 299, Junio 1999, pp. 42-51.

[8] W. Mühlegger, "Identifying Distorting Sources on the Grid”, PCIM 2002, Nuremberg, pp. 45-49.

[9] E. Handschin, W. Horenkamp, Th. Wiesner and E. Stachorra, "The Spectral Grid Impedance of Distribution Networks as a Key Term Analysis", PCIM 2001, Nuremberg, pp. 171-176.

[10] M. Herfurth, "Active Harmonic Filtering for Line Rectifiers of Higher Output Power", Siemens Components, Vol. XXI, ${ }^{\circ}{ }^{1}$, pp. 9-13.

[11] A. K. Lefedjiev and J. Leisten, "Voltage Tracking PFC' A Simple, Low Cost Way to Improve The Efficiency of PC Power Supplies with Active PFC", PCIM 2001, Nuremberg, pp. 95-100.

[12] D. Marsh, "Active Power Factor Correction", EDN Magazine, Jan. 2000, pp. 31-41.

[13] N. G. Hingorani and L. Gyugyi, "Understanding Facts: Concepts and Technology of Flexible AC Transmission Systems", IEEE Press, Piscataway, (2000).

[14] H. Fujita and H. Akagi, "The Unified Power Flow Conditioner: The Integration of Series- and Shunt-Active Filters", IEEE Transactions on Power Electronics, Vol.13, No2, pp.315-322, 1998. 
[15] S. Buso, L. Malesani and P. Mattavelli, "Comparison of Current Control Techniques for Active Filter Applications", IEEE Transactions on Industrial Electronics, Vol.45, № 5, pp.722-729, 1998.
[16] C. Schauder and H. Mehta, "Vector Analysis and Control of Advanced Static VAR Compensators", IEE Proceedings, Part C, Vol.140, Nº 4, pp.299-306, 1993.

[17] T. J. E. Miller, "Reactive Power Control in Electric Circuits", John Wiley and Sons, Toronto, (1982). 